\title{
VIOLENCIA HACIA LAS MUJERES: UN PROBLEMA ESTRUCTURAL
}

Sandra Palestro Contreras y María Soledad Rojas Bravo 


\section{SANDRA PALESTRO CONTRERAS}

Feminista, socióloga, integrante de la Coordinación Nacional de la Red Chilena contra la Violencia hacia las Mujeres.

\section{MARÍA SOLEDAD ROJAS BRAVO}

Activista feminista, apóstata, integrante de la Coordinación Nacional de la Red Chilena contra la Violencia hacia las Mujeres. 


\section{VIOLENCIA HACIA LAS MUJERES: UN PROBLEMA ESTRUCTURAL}

\section{EL MOVIMIENTO DE MUJERES Y FEMINISTA EN LOS ‘80}

La Red Chilena contra la Violencia hacia las Mujeres es parte de un proceso desarrollado por las mujeres en diferentes países de América Latina en la década de los '80. En Chile, durante la dictadura cívico-militar se constituyeron diversas organizaciones sociales, políticas y culturales de mujeres, que contaron entre sus antecedentes los caminos abiertos por sus antecesoras sufragistas y por las obreras de comienzos del siglo XX. En tiempos dramáticos en que asesinatos, detenciones, tortura, desaparición de personas y exilio cimentaban el "nuevo orden", tempranamente las mujeres se fueron organizando en medio del dolor y el duelo.

Las primeras iniciativas de organizaciones de derechos humanos y subsistencia, así como el posterior surgimiento de múltiples organizaciones desde distintas vertientes, fueron mostrando la decisión y capacidad de las mujeres para enfrentar problemas concretos provocados por la dictadura y luego ir ampliando la reflexión hacia el cuestionamiento del sistema político.

En los años '76, '77 y '78 -explicaba Julieta-, los grupos políticos de oposición y democráticos empiezan a repensar, a cuestionar críticamente el sentido y los contenidos de lo que era la democracia. Nosotras, en esos pequeños grupos de mujeres, nos hicimos la pregunta ¿qué significa la democracia para nosotras las mujeres? Es decir, ¿de qué libertad, de qué igualdad y de qué fraternidad se estaría tratando para las mujeres? Entonces empezamos a constatar que había tremendas diferencias entre estos postulados teóricos y la vida concreta que realizábamos las mujeres. La comparación entre estas igualdades formales que se postulaban y las vidas concretas nos llevó a una tremenda rebeldía, a la pregunta de por qué aquello que se dice no lo practicamos, no se ha practicado respecto a nosotras también. Y esta rebeldía nos llevó al feminismo; el feminismo es esta rebeldía por estas tremendas diferencias entre lo que se postula para todo el género humano y lo que vivenciamos concretamente las mujeres ${ }^{1}$.

1. Seminario organizado por FLACSO en Santiago, según grabación sin fecha. En: Gaviola, Largo, Palestro. Una Historia Necesaria. Pp.96-97. 
"Democracia en el país y en la casa" fue un eslogan que, de algún modo, derribaba la división entre lo público y lo privado y que sintetizó las exigencias a la futura democracia.

Problemas como el aborto y el divorcio, salas cunas sin discriminación entre mujeres y hombres, y el acoso sexual, instalados en el debate por las feministas, provocaron graves tensiones y conflictos en los Encuentros Nacionales de la Mujer convocados por el Departamento Femenino de la Coordinadora Nacional Sindical en los años '78, '79 y '80.

Las organizaciones de mujeres fueron confluyendo en articulaciones cada vez mayores. El Departamento Femenino de la Coordinadora Nacional Sindical (CNS) incluyó a sindicalistas y a mujeres de federaciones campesinas; el Movimiento Pro Emancipación de la Mujer Chilena (MEMCH '83) reunió a organizaciones de mujeres de partidos políticos de izquierda e independientes; Mujeres por la Vida convocó a la realización de acciones masivas y de gran visibilidad pública a un amplio espectro de mujeres; organizaciones de sectores poblacionales se agruparon en distintas modalidades, tales como el Movimiento de Mujeres Pobladoras (MOMUPO), entre otras; más tarde, mujeres de partidos de oposición e independientes se agruparon en la Concertación de Mujeres por la Democracia. También en 1983, Julieta Kirkwood, junto a otras feministas, crean la Casa de la Mujer La Morada y realizan su primera acción pública desplegando el lienzo "Democracia Ahora. Movimiento Feminista" en las escalinatas de la Biblioteca Nacional.

A nivel internacional, en 1979 se aprobaba la Convención sobre la Eliminación de Todas las Formas de Discriminación contra la Mujer (CEDAW) y se realizaban las conferencias mundiales convocadas por la ONU en el marco del Decenio de la Mujer. Estas tuvieron lugar en Ciudad de México (1975), Copenhague (1980), Nairobi (1985) y Beijing (1995). Por su parte, la Conferencia Mundial de Derechos Humanos realizada en Viena (1993) reconoció la violencia contra las mujeres como una grave violación a sus derechos humanos, tanto en la vida pública como privada, destacando la responsabilidad de los Estados en prevenir y erradicar la violencia contra las mujeres.

A nivel continental, los Encuentros Feministas Latinoamericanos y del Caribe revelaron la común posición de las mujeres en contextos de regímenes autoritarios, estimularon los vínculos entre ellas y reflejaron la potencia del feminismo de la época. La violencia contra las mujeres fue uno de los ejes principales en el primer encuentro en Bogotá, Colombia, en 1981. Allí se instauró el 25 de noviembre como Día Internacional contra la Violencia hacia las Mujeres, a petición de la delegación dominicana en homenaje a las hermanas Mirabal, y se acordó realizar manifestaciones en todo el continente. 
Desde 1983, las articulaciones de organizaciones comenzaron a elaborar demandas a la democracia ${ }^{2}$ con propuestas en todos los ámbitos, dejando de manifiesto que estas eran imposibles de concretar bajo el régimen cívico militar. La lucha antidictatorial era prioritaria.

La violencia contra las mujeres y el control de su sexualidad y reproducción son asuntos medulares en la dominación de las mujeres que las feministas visibilizaron en este periodo. Respecto a sexualidad y reproducción, el MEMCH '83 incorporó en sus demandas "Respetar y asegurar a las mujeres el derecho a conocer y a decidir sobre su propio cuerpo" y "Asumir un programa de acciones conducentes a la despenalización del aborto" (1985). Del mismo modo, organizaciones que conformaron el movimiento feminista expresaron "Que se respete nuestra libertad de elegir el ser madres o no y que se garantice a mujeres y hombres el derecho a decidir libre y responsablemente el número de hijos, el intervalo de nacimientos y acceso a la información, educación y medios que permitan el ejercicio de este derecho" (Movimiento Feminista, 1988).

En 1989, año en que la dictadura cívico-militar derogó la ley de aborto terapéutico, grupos de trabajo relacionados con la salud de las mujeres y organizaciones sociales y feministas constituyeron el Foro-Red de Salud y Derechos Sexuales y Reproductivos, que vendría a cuestionar la visión hegemónica de salud centrada en lo biomédico junto con promover el autocuidado y la autoayuda entre las mujeres.

Respecto de la violencia contra las mujeres, las articulaciones de organizaciones coincidieron en que debía ser un problema a encarar por el Estado y por toda la sociedad: "Se hace imperativo impulsar auténticas transformaciones en la cultura y en las costumbres, en el marco de una sociedad profundamente democrática" (Manifiesto Feminista, 1983). "Educar a ambos sexos para la práctica de relaciones igualitarias, llevando la democracia al seno de la familia para eliminar toda forma de violencia doméstica y sexual y los malos tratos a las mujeres” (MEMCH '83, 1985, Pliego de las Mujeres presentado a la Asamblea de la Civilidad, 1986, y Movimiento Feminista, 1988). "Se penalice todo acto de discriminación o violencia en contra de la mujer, al igual que cualquiera contravención que impide el libre y pleno ejercicio

2. Manifiesto Feminista. Demandas Feministas a la Democracia, diciembre 1983; Principios y reivindicaciones que configuran la plataforma de la mujer chilena (Coordinadora de Organizaciones Femeninas MEMCH ‘83, 1985); Pliego de las Mujeres (Documento presentado a la Asamblea de la Civilidad, 1986); La Demanda de la Mujer Rural. Resoluciones del Primer Encuentro de la Mujer Rural realizado en julio de 1986 por el Departamento Femenino de la Comisión Nacional Campesina (CNC); A las mujeres chilenas. Movimiento Feminista, 1988; Tramas para un nuevo destino. Propuestas de la Concertación de Mujeres por la Democracia, 1990. 
de sus derechos como persona" (Movimiento Feminista, 1988). "Debe eliminarse todo tipo de violencia doméstica o sexual contra la mujer" (La Demanda de la Mujer Rural, 1986).

Ya en esa época se planteaba la necesidad de efectuar cambios en el ámbito educacional que, hoy es claro, es uno de los muchos donde se manifiesta violencia simbólica hacia las mujeres: "Se impone revisión total de textos escolares, especialmente los de socialización temprana" (Manifiesto Feminista, 1983). "Revisar los textos de educación escolar de modo de no repetir las pautas jerárquicas y autoritarias en la socialización de los roles de la mujer y el hombre" (Concertación de Mujeres por la Democracia, 1990).

Así, con la experiencia organizativa y de acción política históricamente acumulada, con todo el conocimiento producido en intensos debates durante 17 años de dictadura, con la promesa de que "la alegría ya viene" y con este gabinete ministerial, se inauguró la recuperada democracia.

\section{Primer Gabinete Ministerial (1990)}

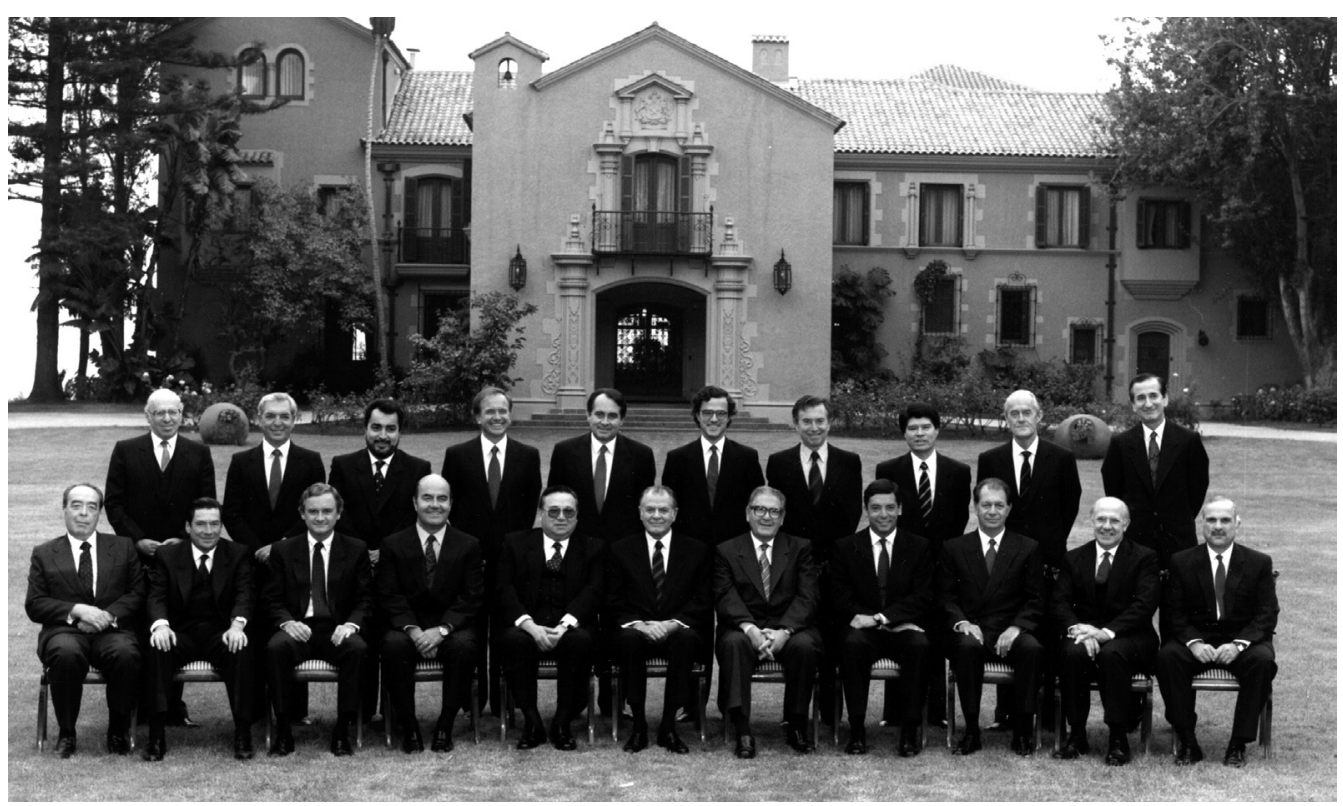

Fuente: https://es.wikipedia.org/wiki/Patricio_Aylwin

\section{RED CHILENA CONTRA LA VIOLENCIA DOMÉSTICA Y SEXUAL}

Como resultado de intensos procesos organizativos, de debate y grandes movilizaciones de las mujeres en los años ‘80, surge en 1990 la Red Chilena contra la 
Violencia Doméstica y Sexual como "un espacio flexible y de construcción colectiva integrado por organizaciones sociales de mujeres, organismos no gubernamentales y personas naturales, todas vinculadas al abordaje de la violencia hacia las mujeres"3. Su finalidad iba más allá de la coordinación de acciones, pues se asumió como "un espacio destinado a la reflexión, intercambio y reciclaje de experiencias en función de instalar las demandas y propuestas de las mujeres en la escena pública"4. Entre sus integrantes se encontraban las primeras instalaciones de acogida para mujeres en riesgo vital organizadas por las propias mujeres, tales como la Casa Mirabal en Coronel, región del Biobío, y la Casa Yela en Talca, región del Maule.

En 1991 se promulgó la ley 19.023 que creó el Servicio Nacional de la Mujer (SERNAM), con rango ministerial, primera institución mandatada para generar políticas públicas para las mujeres. La Red Chilena fue invitada por el naciente servicio a participar, en representación de la sociedad civil, en la Comisión Interministerial Nacional de Prevención de la Violencia Intrafamiliar durante el periodo 1992-1993.

En estos años, las organizaciones de mujeres ejercieron presión en el Parlamento para la ratificación de la Convención Interamericana para Prevenir, Sancionar y Erradicar la Violencia contra la Mujer (Belém do Pará), la que había sido adoptada por la Organización de Estados Americanos (OEA) en 1994. Este hito se concretó en 1996 y dos años después se lograría su promulgación y publicación ${ }^{5}$. La Convención, que posee fuerza de ley para los países, constituía (y constituye) un importante mandato para enfrentar la violencia contra las mujeres. Sin embargo, hasta hoy el Estado chileno no la ha respetado, restringiendo su legislación y política pública al ámbito privado, como violencia intrafamiliar, sin nombrar a las mujeres y a las niñas como las principales afectadas por esta violencia específica.

3. Información contenida en el documento "Campaña contra el Femicidio: Por la vida de las mujeres: Ni una muerte más. 25 de noviembre: Día Internacional contra la Violencia hacia las Mujeres", 2001.

4. Ibidem, sin número de página.

5. Convención Belem do Pará. Artículo 1. Para los efectos de esta Convención debe entenderse por violencia contra la mujer cualquier acción o conducta, basada en su género, que cause muerte, daño o sufrimiento físico, sexual o psicológico a la mujer, tanto en el ámbito público como en el privado. Artículo 2. Se entenderá que violencia contra la mujer incluye la violencia física, sexual y psicológica: a. que tenga lugar dentro de la familia o unidad doméstica o en cualquier otra relación interpersonal, ya sea que el agresor comparta o haya compartido el mismo domicilio que la mujer, y que comprende, entre otros, violación, maltrato y abuso sexual; b. que tenga lugar en la comunidad y sea perpetrada por cualquier persona y que comprende, entre otros, violación, abuso sexual, tortura, trata de personas, prostitución forzada, secuestro y acoso sexual en el lugar de trabajo, así como en instituciones educativas, establecimientos de salud o cualquier otro lugar; y c. que sea perpetrada o tolerada por el Estado o sus agentes, donde quiera que ocurra. 
Cuando fue promulgada la ley 19.325 sobre Violencia Intrafamiliar (VIF, 1994), la Red Chilena se propuso dar seguimiento a su implementación y evidenciar la insuficiencia del concepto VIF para dar cuenta de la violencia contra las mujeres, tanto al interior de la Comisión Interministerial como en la sociedad en general. A poco andar y durante años, la Red Chilena contra la Violencia Doméstica y Sexual junto a otras organizaciones de mujeres fueron evidenciando sus consecuencias.

“... tanto en relación a la invisibilización conceptual de la violencia contra las mujeres -a la que corresponde una aplastante mayoría de los casos de VIFcomo en cuanto a las consecuencias de la adopción de políticas públicas basadas en los conceptos dados por la ley, lo cual instaló a la violencia intrafamiliar en el plano social y político como concepto de fondo, como el fenómeno contra el cual se organiza el aparato público y la reacción social (Toledo, p.13)”’6.

La Ley sobre Violencia Intrafamiliar encubre las jerarquías de poder existentes al interior de las familias y las relaciones de pareja, e invisibiliza que estas están presentes en todos los ámbitos sociales, despolitizando el problema.

La Ley VIF se modificó en 2005, en momentos en que culminaba la instalación de la Reforma Procesal Penal y la creación de los Tribunales de Familia. En el marco de esa reforma se tipifica un nuevo delito, el de "maltrato habitual", el que debía ser calificado por un Tribunal de Familia antes de ser ingresado al sistema penal, lo cual constituyó una nueva traba para las mujeres en el acceso a la justicia. Por otra parte, la habitualidad del maltrato es una extraña concepción del delito, puesto que es el único que debe ser reiterado para calificar como tal. En 2012, el Comité de la CEDAW exhortó al Estado chileno a que "elimine el delito de maltrato habitual a fin de que puedan realizarse investigaciones penales de todos los actos de violencia doméstica y se procese a los autores"ᄁ. Así también, a que promulgara legislación para tipificar como delito el acoso sexual.

6. Toledo Vásquez, Patsilí. Introducción. En: Tipificación del femicidio en Chile. Un debate abierto. Red Chilena contra la Violencia hacia las Mujeres. 2009. pp.13-23.

7. Comité para la Eliminación de la Discriminación contra la Mujer. Observaciones finales sobre los informes periódicos quinto y sexto de Chile, adoptadas por el Comité en su $53^{\circ}$ periodo de sesiones ( $1^{\circ}$ a 19 de octubre de 2012). 


\section{REPOLITIZAR LA VIOLENCIA CONTRA LAS MUJERES}

A partir del conocimiento de los masivos asesinatos de mujeres en Ciudad Juárez, México, y en Guatemala, la Red Feminista Latinoamericana y del Caribe contra la Violencia Doméstica y Sexual, de la cual formó parte la Red Chilena, acordó desarrollar la campaña "Por la vida de las mujeres. Ni una muerte más" (2000). Al mismo tiempo, promovió la investigación y denuncia de estos hechos extremos de violencia en cada uno de los países, identificando y nombrando el femicidio ${ }^{8}$.

En esa perspectiva, la Red Chilena publicó en 2004 la investigación Femicidio en Chile ${ }^{9}$, instalando en el país un concepto que evidenció los asesinatos de mujeres en razón de su condición de género como actos extremos de poder, dominio y control sobre sus vidas, naturalizados y tolerados social e institucionalmente. El concepto femicidio señala con claridad lo que el término "violencia intrafamiliar" había encubierto, que las "víctimas" son mujeres.

El estudio estableció una relación directa entre violencia intrafamiliar y femicidio, nexo no considerado en el propio sistema judicial. La nueva Ley VIF en discusión debía considerar esta relación, por lo que, a petición del Subsecretario del Interior, la Red Chilena participó en la elaboración de indicadores de riesgo vital para introducir en el parte policial de las denuncias de VIF, en conjunto con la Dirección de Protección Policial de la Familia (DIPROFAM), repartición perteneciente a Carabineros de Chile, y Seguridad Ciudadana.

En 2005, la Red Chilena organizó y convocó la primera manifestación coordinada y simultánea en ocho regiones del país. Una marcha nocturna con antorchas en conmemoración del 25 de noviembre, Día Internacional contra la Violencia hacia las Mujeres. Ese mismo año, ante la inexistencia de información consolidada, se propuso compilar la oferta institucional de programas y servicios para mujeres que viven violencia en el ámbito doméstico en seis regiones del país, así como mapear las organizaciones sociales de mujeres y feministas presentes en las respectivas localidades. Esto, en el contexto de un análisis crítico hacia las precarias políticas de gobierno en la materia y de un debate que ya había comenzado sobre la necesidad de repolitizar la problemática. Es así como en 2006 la Red publicó la Guía de recursos

8. El concepto femicidio/feminicidio fue acuñado por Diana Russell y Jill Radford en 1992.

9. Rojas Bravo, Soledad (Coord); Maturana Kesten, Camila; Maira Vargas, Gloria. Femicidio en Chile. Corporación La Morada, Red Chilena contra la Violencia Doméstica y Sexual, Naciones Unidas. Santiago, 2004. 89p. 
para la acción ${ }^{10}$, construida colectivamente con las organizaciones en un proceso articulador que fortaleció vínculos y generó un conocimiento necesario para todas.

El delito de femicidio se incorporó a la legislación chilena en 2010, como subcategoría del delito de parricidio; la ley se aprobó en el mismo sentido restrictivo de la VIF. Sólo considera femicidios los asesinatos de mujeres por parte de sus parejas o exparejas, excluyendo los crímenes perpetrados en otros contextos: mujeres y niñas violadas y asesinadas por conocidos, amigos, desconocidos, clientes en el caso de las trabajadoras sexuales, y también los crímenes de odio a lesbianas. A ocho años de tipificado el delito, el actual Ministerio de la Mujer y Equidad de Género (ex SERNAM) registra 325 femicidios, mientras que para la Red Chilena, durante ese periodo han sido asesinadas 456 mujeres dentro del mismo patrón misógino. No obstante la existencia de legislación y política pública, estos crímenes no han disminuido; más aún, un alto porcentaje de las mujeres asesinadas contaba con medidas cautelares.

Diez años después del primer estudio Femicidio en Chile, la Red Chilena realizó una segunda investigación ${ }^{11}$, Violencia extrema hacia las mujeres, que abarca dos años a partir de la tipificación del delito, 2010-2012. Este estudio reveló otras realidades de violencia directamente vinculadas al femicidio, conceptualizadas como suicidio femicida, referido a mujeres que se quitan la vida producto de la extrema violencia machista que las afecta, y castigo femicida, también conocido en otros países como femicidio vinculado o por conexión familiar, que identifica el asesinato a personas que forman parte del vínculo afectivo de la mujer, principalmente hijas e hijos, con el objetivo de destruirla psicológicamente. El estudio también relevó que los femicidios frustrados corresponden al doble de los consumados, lo que significa que además de las mujeres asesinadas cada año, muchas sobreviven y sufren ese daño toda su vida.

En las últimas décadas se han promulgado leyes que mitigan o eliminan inequidades, pero la trama cultural androcéntrica es fuerte e impone sus normas. "En 2008, la fuerte arremetida del conservadurismo católico y fundamentalista desde sus plazas políticas en la UDI y el Opus Dei- refuerza las políticas de control y restringe aún más la libertad de las mujeres. Por la vía de la denegación institucional de la anticoncepción de emergencia en un fallo del Tribunal Constitucional, se niega

10. Red Chilena contra la Violencia Doméstica y Sexual. Guía de Recursos para la Acción, 2006. Participaron Solidaridad y Organización Local (SOL), Casa de la Mujer Yela, Centro de Atención y Apoyo a Víctimas de Violencia Mirabal y colectivos feministas, organizaciones todas ellas articuladas en la Red.

11. Santana Nazarit, Paula; Astudillo Pérez, Lorena. Violencia extrema hacia las mujeres en Chile (2010-2012). Red Chilena contra la Violencia hacia las Mujeres. Santiago, 2014.162p. 
a las mujeres el ejercicio libre de su sexualidad y el control de su reproducción"12. La reciente promulgación de la ley sobre aborto (2017), retrasada por décadas en el Parlamento, contempla sólo tres situaciones extremas en la vida de las mujeres para interrumpir un embarazo: riesgo de vida de la mujer, inviabilidad fetal y violación. Estas tres causales responden a aproximadamente un 3\% de las mujeres que abortan, es decir, se mantiene la criminalización, exponiendo a las mujeres a efectuar estos procedimientos en clandestinidad y riesgo para sus vidas.

La precariedad de la legislación referida a la violencia hacia las mujeres se expresa también en la Ley sobre Acoso Sexual, que restringida al ámbito laboral, sólo contiene sanciones administrativas y entrega al empleador que recibe la denuncia la facultad de optar entre hacer una investigación interna o derivarla a la Inspección del Trabajo, en circunstancias de que en la mayoría de los casos es el mismo empleador o un superior jerárquico el agresor. Se sabe, por evaluaciones de la ley, que la mayoría de las mujeres, generalmente jóvenes, terminó renunciando al trabajo. Por su parte, la ley sobre igualdad de remuneraciones, que buscaba cumplir con la implementación del convenio $\mathrm{N}^{\mathrm{0}} 100$ de la OIT sobre igualdad de remuneración por trabajo de igual valor, fue cambiada en el Parlamento por igual remuneración por un mismo trabajo, lo que abrió las puertas a su vulneración. Una evaluación realizada a cinco años de su puesta en vigencia concluye que no ha cumplido su objetivo.

Un estudio realizado por Corporación Humanas y el Observatorio Derechos Humanos UDP ${ }^{13}$ reveló que la violencia sexual, sistemáticamente infringida a mujeres prisioneras políticas durante la dictadura cívico-militar, llegó a conocimiento de los tribunales de justicia y que fue mayoritariamente invisibilizada, lo que permitió que estos crímenes permanecieran en total impunidad. Las leyes nacionales consideran la violencia sexual como "un daño colateral" en la tortura. Mujeres sobrevivientes, algunas agrupadas en el "Colectivo Mujeres Sobrevivientes Siempre Resistentes" y con el apoyo de organizaciones de derechos humanos y de mujeres, entre ellas la Red Chilena, trabajaron arduamente y sin resultados por la tipificación de la violencia política sexual como crimen de lesa humanidad.

12. Rojas Bravo, Soledad. " $¡$ Cuidado! El machismo mata”. Resistencia de las mujeres a la violencia. En: Nación golpeadora. Manifestaciones y latencias de la violencia machista. Red Chilena contra la Violencia Doméstica y Sexual, 2000. p.20.

13. Maturana, Camila y Quintanilla, Daniela; Zamorano, Paulina y González, M. Florencia. Respuesta judicial a la violencia sexual contra las mujeres en dictadura. Corporación Humanas y Observatorio Derechos Humanos UDP. Santiago, 2013. 


\section{RED CHILENA CONTRA LA VIOLENCIA HACIA LAS MUJERES}

El énfasis puesto desde comienzos de los '90 en las demandas hacia el Estado para la protección de las mujeres y la erradicación de la violencia ya venía siendo cuestionado. La identificación del femicidio como expresión extrema del ejercicio de poder machista y la inexistencia de un concepto de violencia hacia las mujeres en la legislación y políticas implementadas -con su consecuente ineficacia y victimización de las mujeres- impulsan a la Red Chilena a reorientar su acción hacia el fortalecimiento de las organizaciones y espacios de articulación como estrategia de poder. En esta línea, se propone hacer alianzas con otros sectores y movimientos sociales afectados por la discriminación y la exclusión.

En el contexto de la reorientación de su política, la Red Chilena desarrolla a partir de 2007 la campaña " $C$ Cuidado! El machismo mata"14. La identificación del femicidio, la violencia sexual y la violencia simbólica son los ejes articuladores de la campaña, que tiene como propósito establecer las conexiones entre sus distintas manifestaciones. La calle es el lugar principal para su puesta en escena y las organizaciones de mujeres, su principal soporte y medio de circulación.

La instalación del Memorial itinerante a las víctimas de femicidio en Chile, simbolizadas en cientos de pares de zapatos con el nombre de la mujer asesinada, su edad y relación con el femicida, dio inicio a la campaña.

Las organizaciones articuladas en la Red Chilena convocan a las mujeres en todo el país a erradicar la violencia de nuestras vidas; a rechazar pública y privadamente toda forma de agresión machista; a emplazar a todos los sectores sociales y políticos a no ser cómplices, repudiando el femicidio; y a exigir de parte de las instituciones públicas, políticas acordes a la gravedad del problema: protección eficaz, servicios de calidad, justicia y reparación. No más víctimas sino mujeres con autonomía para decidir sobre sus vidas y derechos garantizados (Rojas, 2000, p.20) ${ }^{15}$.

De Arica a Punta Arenas, organizaciones de mujeres y feministas se reúnen para discutir y coordinar acciones callejeras. Hacen visibles el abuso y la violencia contra mujeres y niñas, y la impunidad; organizan grupos de formación y debate, y generan variadas formas comunicacionales. Cientos de mujeres se suman a las acciones e interpelan a la sociedad en general, a los medios de comunicación y a las autoridades en su responsabilidad de actuar contra la violencia hacia las mujeres.

14. Eslogan y diseño de autoría de la española Ángeles Álvarez, que une magistralmente en cuatro palabras la relación entre el femicidio y la cultura patriarcal machista que lo sostiene. Sobre este diseño, la Red Chilena desarrolla su campaña “¡Cuidado! El machismo mata” desde 2007 hasta la fecha.

15. Rojas, Soledad. op.cit. 
Durante doce años (2007-2018) la campaña “¡Cuidado! El machismo mata” ha sido una importante herramienta articuladora de las organizaciones que integran la Red Chilena y más allá de estas, interesa a mujeres que trabajan en oficinas comunales y/o centros de la mujer que se identifican con los mensajes de la campaña y solicitan afiches para su difusión. Estos constituyen un importante apoyo para la reflexión colectiva, que ha contribuido a la desnaturalización de la violencia machista y a la generación de conciencia respecto de su necesaria erradicación. Una gran fortaleza de la campaña es su elaboración colectiva por parte de las organizaciones de mujeres que constituyen la Red Chilena en todo el país, quienes año a año le imprimen pertinencia nacional y local.

La generación de conocimiento es un propósito permanente de la Red Chilena, que aporta a la identificación de la violencia patriarcal como factor clave en la sujeción de las mujeres, promueve la reflexión feminista, nutre el activismo político de las organizaciones que la integran y abre espacios a la circulación de la palabra de las mujeres.

Entre sus publicaciones ${ }^{16}$ se encuentran el estudio Violencia sexual y aborto $(2008)^{17}$, que pone al descubierto la relación entre ambos, situando la problemática en el contexto más amplio de denegación de la libertad sexual y reproductiva de las mujeres; Tipificación del femicidio en Chile (2009), una compilación de ponencias del seminario homónimo en medio del debate parlamentario por la tipificación del delito; Nación golpeadora. Manifestaciones y latencias de la violencia machista (2009); Mujeres y violencia: silencios y resistencias (2013); y El continuo de violencia hacia las mujeres y la creación de nuevos imaginarios (2015), publicaciones que contienen artículos de diferentes autoras que reflexionan y profundizan sobre la violencia patriarcal. En cada una de ellas participaron integrantes, colaboradoras y amigas de la Red Chilena, las que con miradas críticas y profundas sobre los asuntos que tratan, han contribuido a la ampliación de la conciencia feminista.

La formación feminista es clave tanto para la producción de nuevos contenidos como para la elevación de la conciencia de las mujeres respecto de la opresión, discriminación y violencia, y condición necesaria para la transformación social. En esta línea, la Red Chilena -las organizaciones que la integran en las diferentes regiones del país- realiza habitualmente conversatorios, seminarios o jornadas de reflexión en las que participan mujeres de la Coordinación Nacional y de otras

16. Disponibles en http://www.nomasviolenciacontramujeres.cl/publicaciones/

17. Maira Vargas, Gloria; Santana Nazarit, Paula; Molina Sáez, Siomara. Violencia Sexual y Aborto. Conexiones necesarias. Red Chilena contra la Violencia Doméstica y Sexual. Santiago, 2008. $143 \mathrm{p}$. 
regiones. La producción de cartillas de información y formación feminista ${ }^{18}$ y la sistematización de las experiencias de escuelas implementadas por organizaciones de mujeres en diferentes regiones son una constante en el trabajo de la Red Chilena. Estudiantes en práctica y tesistas de diversas universidades aportan a la generación de conocimiento, a la vez que se nutren de la reflexión cotidiana y la participación en acciones públicas.

La articulación y la reflexión conjunta posibilitan la actualización de contenidos y propuestas de la Red Chilena en medio de los procesos sociales en curso. Los debates y reflexiones se van enfocando hacia factores clave en la producción y reproducción de la violencia práctica y simbólica hacia las mujeres, principalmente en los medios de comunicación, la publicidad y la educación. Respecto de los medios de comunicación y la publicidad, la Red Chilena ha contribuido al conocimiento, visibilidad y denuncia de sus manifestaciones sexistas. En cuanto a la educación, a partir de 2011, durante la irrupción de los movimientos sociales liderados por el movimiento estudiantil, la Red Chilena incorporó a la demanda de educación pública y gratuita que esta fuera laica y no sexista. En consecuencia, inicia una línea de acción en este ámbito específico con una revisión crítica de los textos escolares. En 2016 publica Educación No Sexista. Hacia una Real Transformación ${ }^{19}$, libro en el que se expone tanto el sexismo que impregna todo el sistema educativo y sus efectos en la vida de las mujeres como las experiencias de educación no sexista desarrolladas por feministas a través de módulos, cursos y talleres en escuelas y liceos en distintos territorios del país.

La omisión de las mujeres en el relato histórico y la construcción de un estereotipo femenino, ya evidenciadas en estudios anteriores, continúa reproduciéndose en los textos de todas las asignaturas. En el contexto de las actuales movilizaciones estudiantiles que denuncian el sexismo en la educación, el estudio de la Red Chilena cobra mayor relevancia. La falta de referencias para las mujeres, tanto individuales como colectivas, llevó a la Red Chilena a buscar en la historia explicaciones de tal fenómeno. Con este propósito, convocó a docentes e historiadoras feministas a una reflexión que dio como resultado el texto Nunca más mujeres sin historia ${ }^{20}$.

La reflexión desarrollada por la Red Chilena se nutre de y nutre a la vez el activismo político. Las asambleas nacionales y encuentros regionales son instancias

18. Disponibles en www.nomasviolenciacotramujeres.cl

19. Red Chilena contra la Violencia hacia las Mujeres. Educación no sexista. Hacia una real transformación. Santiago, 2016. 132p.

20. Red Chilena contra la Violencia hacia las Mujeres. Palestro Contreras, Sandra; Jamett Pizarro, Francia; Lillo Muñoz, Daniela; Lopresti Martínez, Lorella; Toro Céspedes, María Stella. Nunca más mujeres sin historia. Conversaciones feministas. Santiago, 2018. 153p. 
que permiten el análisis de la situación nacional, la retroalimentación de experiencias y la planificación de acciones conjuntas. El intercambio que se produce en estas instancias da cuenta del quehacer de las organizaciones y su participación en distintos movimientos comunales y territoriales en sus localidades respectivas. La Campaña "¡Cuidado! El machismo mata", su inicio a mediados del año y su cierre el 25 de noviembre, es la principal acción coordinada en todo el país.

La Red Chilena no cuenta con servicios de atención para mujeres que viven violencia -esta es una responsabilidad de la institucionalidad estatal-, pero orienta a las mujeres que consultan, derivándolas a los servicios de atención disponibles. No obstante, en algunas circunstancias específicas acompaña a mujeres agredidas o a familiares de mujeres asesinadas.

Al importante posicionamiento público de la Red Chilena han contribuido tanto sus reflexiones y acciones a nivel regional y nacional como un trabajo comunicacional que utiliza diferentes medios y formatos, tales como vocerías, medios virtuales, redes sociales y otros; la producción de un boletín mensual de noticias y un dossier anual con información actualizada sobre violencia hacia las mujeres que se distribuye a las organizaciones sociales y medios de comunicación. Asimismo, produce el programa Mujeres en Sintonía para radios comunitarias.

\section{AFIRMAR NUESTRA AUTONOMÍA}
"El feminismo, junto con ser un cuerpo de conocimientos, es acción transformadora de la sociedad. Hay así una relación dialéctica: el meterse en el mundo como mujeres conscientes de su condición, es una acción transformadora del mundo y esto es la praxis feminista (entrar transformando)" (Kirkwood, p.108) $)^{21 .}$

Los movimientos feministas en el país y en el continente -de los que la Red Chilena forma parte- han contribuido al desarrollo del feminismo en su teoría y su práctica.

La violencia contra las mujeres es violencia específica, arraigada en la cultura y naturalizada en el sentido común; responde, en todas sus expresiones, a un patrón cultural androcéntrico y de dominación masculina, presente estructuralmente en la organización del Estado, la sociedad y el mercado, transmitido por todas las

21. Kirkwood, Julieta. Feminarios. Ediciones Documentas. Santiago, 1987. 
instancias de socialización. La identificación de un patrón común permite establecer conexiones entre sus distintas manifestaciones.

La errada conceptualización del problema de violencia hacia las mujeres por el Estado chileno, que la entiende como asunto de familia o de pareja, se refleja en la parcialidad de la legislación y las políticas públicas. El abordaje institucional es asistencialista y fragmentado, lo que genera dependencia y revictimización, ocultando su carácter político en tanto asunto medular de la discriminación y opresión de las mujeres.

La actual visibilidad de los movimientos feministas y de las transformaciones que se proponen da cuenta de un proceso de desarrollo de la conciencia en las mujeres que conlleva al creciente cuestionamiento de las estructuras de dominación patriarcal.

Las tomas feministas de liceos y universidades, las movilizaciones callejeras contra la violencia hacia las mujeres, por una educación no sexista, por el aborto libre, por la identidad de género, muestran la creciente politización de las experiencias inscritas en los cuerpos subordinados y desenmascaran las relaciones de poder y las prácticas que las reproducen y naturalizan. Son estas "mozas insolentes" las que ahora se hacen oír. "Elegir entre la mesura y la insolencia tiene que ver con estrategias políticas", sostiene Julieta Kirkwood.

En este contexto se han generalizado términos tales como feminismo, patriarcado y sexismo, en los que es necesario profundizar dado que sus contenidos han entrado en un peligroso terreno de disputa y neutralización por el oportunismo político de sectores conservadores interesados en mantener el orden vigente.

Afirmar la autonomía de las mujeres, elevar la conciencia social sobre la necesidad de erradicar la violencia patriarcal, trabajar por la confluencia de los feminismos y con diversos movimientos sociales son desafíos actuales para la Red Chilena contra la Violencia hacia las Mujeres en la perspectiva de socavar las estructuras de dominación. 


\section{REFERENCIAS}

Gaviola, Edda; Largo, Eliana; Palestro, Sandra. Una historia necesaria. Mujeres en Chile: 1973-1990. Edición propia, Santiago, 1994.

Maira Vargas, Gloria; Santana Nazarit, Paula; Molina Sáez, Siomara. Violencia Sexual y Aborto. Conexiones necesarias. Red Chilena contra la Violencia Doméstica y Sexual. Santiago, 2008.

Maturana, Camila y Quintanilla, Daniela; Zamorano, Paulina y M. Florencia González. Respuesta judicial a la violencia sexual contra las mujeres en dictadura. Corporación Humanas y Observatorio Derechos Humanos UDP. Santiago, 2013.

Red Chilena contra la Violencia Doméstica y Sexual, 2000. Nación golpeadora. Manifestaciones y latencias de la violencia machista. Santiago, 2000.

Red Chilena contra la Violencia Doméstica y Sexual. Guía de Recursos para la Acción. Santiago, 2006.

Red Chilena contra la Violencia Doméstica y Sexual. Tipificación del femicidio en Chile. Un debate abierto. Santiago, 2009.

Red Chilena contra la Violencia hacia las Mujeres. Educación No Sexista. Hacia una Real Transformación. Santiago, 2016.

Red Chilena contra la Violencia hacia las Mujeres. Palestro Contreras, Sandra; Jamett Pizarro, Francia; Lillo Muñoz, Daniela; Lopresti Martínez, Lorella; Toro Céspedes, María Stella. Nunca más mujeres sin historia. Conversaciones feministas. Santiago, 2018.

Rojas Bravo, Soledad (Coord); Maturana Kesten, Camila; Maira Vargas, Gloria. Femicidio en Chile. Corporación La Morada, Red Chilena contra la Violencia Doméstica y Sexual, Naciones Unidas. Santiago, 2004.

Santana Nazarit, Paula; Astudillo Pérez, Lorena. Violencia extrema hacia las mujeres en Chile (2010-2012). Red Chilena contra la Violencia hacia las Mujeres. Santiago, 2014. 